\title{
Mutation spectrum of Drosophila CNVs revealed by breakpoint sequencing
}

\author{
Margarida Cardoso-Moreira*, J Roman Arguello and Andrew G Clark
}

\begin{abstract}
Background: The detailed study of breakpoints associated with copy number variants (CNVs) can elucidate the mutational mechanisms that generate them and the comparison of breakpoints across species can highlight differences in genomic architecture that may lead to lineage-specific differences in patterns of CNVs. Here, we provide a detailed analysis of Drosophila CNV breakpoints and contrast it with similar analyses recently carried out for the human genome.

Results: By applying split-read methods to a total of 10x coverage of 454 shotgun sequence across nine lines of D. melanogaster and by re-examining a previously published dataset of CNVs detected using tiling arrays, we identified the precise breakpoints of more than 600 insertions, deletions, and duplications. Contrasting these CNVs with those found in humans showed that in both taxa CNV breakpoints fall into three classes: blunt breakpoints; simple breakpoints associated with microhomology; and breakpoints with additional nucleotides inserted/deleted and no microhomology. In both taxa CNV breakpoints are enriched with non-B DNA sequence structures, which may impair DNA replication and/or repair. However, in contrast to human genomes, non-allelic homologousrecombination (NAHR) plays a negligible role in CNV formation in Drosophila. In flies, non-homologous repair mechanisms are responsible for simple, recurrent, and complex CNVs, including insertions of de novo sequence as large as $60 \mathrm{bp}$.
\end{abstract}

Conclusions: Humans and Drosophila differ considerably in the importance of homology-based mechanisms for the formation of CNVs, likely as a consequence of the differences in the abundance and distribution of both segmental duplications and transposable elements between the two genomes.

Keywords: Copy number variants, CNVs, Non-allelic homologous-recombination, NAHR, Single-strand annealing, SSA, Non-homologous end-joining, NHEJ, Replication-associated repair, Alternative end-joining, Microhomologymediated end-joining, MMEJ, Filler DNA

\section{Background}

One of the most surprising discoveries about genome sequence variation was the finding that copy number variants (CNVs; that is, duplications, deletions, and insertions) are widespread in eukaryotic genomes. CNVs have the potential to create novel genes, to alter gene structures, and/or to change gene regulation. As a result, $\mathrm{CNVs}$ can cause large phenotypic effects, ranging from highly deleterious [1,2], to CNVs underlying adaptation to novel environments [3,4]. The phenotypic effects of CNVs shape their genomic distribution: in natural populations, $\mathrm{CNVs}$ are

\footnotetext{
* Correspondence: mmc256@cornell.edu

Department of Molecular Biology and Genetics, Cornell University, 526 Campus Road, Ithaca, NY 14853-2703, USA.
}

strongly depleted among protein-coding genes and other functional elements of the genome $[5,6]$. However, in addition to selection, mutational processes also impact the genomic distribution of CNVs [7-10]. The distribution of these variants is not uniform across the genome; instead, $\mathrm{CNVs}$ accumulate in discrete regions as a consequence of local increases in the mutation rate. Consequently, current efforts aimed at the identification of the causal CNVs of both deleterious and adaptive phenotypes could be greatly enhanced by a better understanding of the mutational processes underlying the formation of CNVs and the genomic features associated with elevated mutation rates.

CNVs are formed when the repair of DNA breaks (mostly DNA double-strand breaks) is not perfect, leading to the creation of copy-number mutations. DNA double-strand

\section{Ciomed Central}

(c) 2013 Cardoso-Moreira et al.; licensee BioMed Central Ltd. This is an open access article distributed under the terms of the Creative Commons Attribution License (http://creativecommons.org/licenses/by/2.0), which permits unrestricted use, distribution, and reproduction in any medium, provided the original work is properly cited. 
breaks arise as part of the normal metabolism of the cell or as a consequence of ionizing radiation or reactive oxygen species $[11,12]$. There are three molecular pathways available to repair the breaks, two that require sequence homology to perform the repair - homologous recombination (HR) and single-strand annealing (SSA) - and one that is homology-independent - non-homologous end-joining (NHEJ). Although both HR and SSA require sequence homology to repair DNA double-strand breaks, they differ in the extent of homology that is required: 100 to $200 \mathrm{bp}$ for HR versus as little as $50 \mathrm{bp}$ for SSA [11,12]. Another difference is that while SSA always creates a deletion as a consequence of the repair (it is a mutagenic repair pathway), most of the time HR repairs the DNA break without generating any mutation. However, the existence of segmental duplications (also called low copy repeats (LCRs)) or transposable elements near the DNA break can lead to misalignments in the region. In this case, the repair occurs between misaligned repeats leading to the formation of duplications and deletions in a process known as non-allelic homologous recombination (NAHR). In the absence of sequence homology the cell can use non-homologous pathways to repair DNA double-strand breaks. NHEJ, like SSA, is mutagenic, usually resulting in nucleotide substitutions or small indels, but it can also create larger insertions and deletions. While NHEJ does not require sequence homology, a related alternative end-joining pathway, microhomology-mediated endjoining (MMEJ), uses microhomology to mediate the repair [13]. The different molecular pathways are therefore associated with different types of breakpoints and classes of CNVs: NAHR is associated with large stretches of sequence identity and generates both duplications and deletions; SSA is associated with smaller stretches of sequence identity and only generates deletions; NHEJ and its associated pathways (for example, MMEJ) are associated with either presence (2 to $10 \mathrm{bp}$ ) or absence of microhomology, and are mostly associated with deletions and insertions (although it can also generate duplications) [11,12].

In recent years, additional molecular mechanisms have been proposed to operate in association with replicationbased repair and cause CNVs. These mechanisms were proposed following the observation that a subset of human CNVs are highly complex $[12,14,15]$. Such complex CNVs are hard to explain given the canonical HR (and the associated NAHR) and NHEJ pathways because they would require multiple DNA double-strand breaks. Furthermore, the analysis of the breakpoints of these CNVs suggested multiple rounds of strand invasion and the copying of nearby sequences $[12,14,15]$, signatures that could more easily be explained by replication forks stalling (or collapsing), and subsequently disengaging from the template and re-annealing. Three of the proposed models are: fork stalling and replication switching (FoSTeS) [16], microhomology-mediated break-induced replication
(MMBIR) [17], and serial replication slippage (SRS) [18]. Although these models differ in specific details [12,14], they are essentially indistinguishable in terms of breakpoint analysis. They all share the requirement that the reannealing is mediated by microhomology, and they also suggest that templated DNA from nearby sequences can be introduced at the breakpoints $[12,14]$. Although these models have also been proposed to mediate the formation of simple CNVs, it is challenging to distinguish the signatures of these microhomology-mediated replication models from those of NHEJ (and associated MMEJ). In principle, one could distinguish between the two when there are additional nucleotides present at CNV breakpoints: replication-based models would predict that the additional nucleotides correspond to templated DNA (that is, the extra nucleotides were copied from a nearby location) while NHEJ/MMEJ would predict that the additional nucleotides correspond to filler DNA (that is, the extra nucleotides were randomly incorporated).

Most of the work in CNV breakpoint identification has been restricted to mammalian genomes, and in particular to the human genome [19-23]. In humans (as in other mammals) CNVs are significantly enriched close to segmental duplications $[8,12]$. These regions were initially proposed, and subsequently shown to be, CNV hotspots predominantly through facilitating NAHR $[7,8,12]$. However, not all human CNV hotspots are associated with segmental duplications; in fact, a sizeable fraction is not [7,21]. Here, we aim to further our understanding of the mutational mechanisms underlying the formation of CNVs by extending breakpoint analysis to the $D$. melanogaster genome. CNVs are as widespread in the fly as in mammalian genomes $[5,24,25]$, and $\mathrm{CNV}$ hotspots have been identified in both D. melanogaster [9] and its sister species, D. simulans [10]. Although patterns of copy number variation share many similarities between humans and flies, the two genomes have very different genomic architectures. For example, while segmental duplications comprise approximately $5 \%$ of the human and mouse genomes, they comprise only $1 \%$ of the fly genome [26]. Similarly, while transposable elements comprise approximately $50 \%$ of the human genome, they only correspond to $20 \%$ of the fly genome, where they are mostly restricted to pericentromeric regions and the fourth chromosome [27]. (The same holds true for segmental duplications [26].) Our goal was to take advantage of the differences in genome architecture between flies and humans in order to dissect the contribution of different genomic features to the formation of CNVs. We have done this by examining two distinct sets of CNVs: one generated using long Roche/454 sequencing reads [28] and the other using high-resolution tiling microarrays [5]. The use of these two dataset sets has enabled us to overcome many of the potential biases associated with each individual method if used alone. Our results indicate that fly CNVs share 
several of the striking characteristics observed for human CNVs: (1) a paucity of breakpoints associated with both microhomology and additional nucleotides inserted/deleted at the breakpoints; (2) an enrichment of non-B DNA sequences at the $\mathrm{CNV}$ breakpoints; and (3) a significant fraction of both recurrent and complex CNVs. Importantly, however, the different architectural organization of the fly genome does appear to shape patterns of copy number variation: homology-based pathways (notably NAHR) play a minor role in the formation of fly CNVs, including recurrent CNVs. Our data indicate that in flies non-homologous pathways underlie most CNV formation for both simple and complex events. One important consequence is that in flies most insertions do not correspond to duplications of previously existing sequence but are instead created de novo by the random insertion of nucleotides and/or small repeats from nearby sequences.

\section{Results}

\section{Precise breakpoint detection of CNVs from a 454 sequencing dataset}

Sackton and colleagues sequenced at low coverage (approximately $0.2 \mathrm{x}$ ) the genomes of nine $D$. melanogaster strains using Roche/454 technology [28]. These genome sequences were used to evaluate the extent to which population genomic inferences could be made from low/sparse genomic coverage. Sackton and colleagues identified not only SNPs, but also transposable elements and CNVs. However, the latter were identified using a paired-end framework that did not provide the exact breakpoints of the CNVs. Here, we employ a different approach to detect CNVs based on split-read mapping that is capable of detecting CNVs with precise breakpoint resolution (that is, single nucleotide resolution). Defining what is the minimum size of a variant for it to be considered a CNV as opposed to an indel is largely arbitrary and often reflects the degree of resolution of the platform used to identify those variants. While initial CNV studies defined these variants as being at least $1 \mathrm{~kb}$ in length, more recent studies (for example, 1000 Genomes Project [21]) use $50 \mathrm{bp}$ as the lower limit for calling a variant a CNV. In agreement with the previous literature on Drosophila CNVs $[5,10]$, here we use $25 \mathrm{bp}$ as the lower limit to classify insertions, deletions, and duplications as CNVs.

We downloaded the raw data for the nine genomes sequenced by Sackton and colleagues [28] and aligned the reads against the $D$. melanogaster reference genome using the aligner Mosaik [29]. We discarded all reads that mapped to the reference genome and focused only on the subset of the reads that failed to map. We re-aligned these reads to the reference genome using BLAT [30] (see Methods). Because BLAT was designed to align mRNA onto genomic DNA, it does not penalize the existence of large gaps between the reads and the reference genome and provides the exact location of those gaps. By parsing the BLAT results we identified all reads that: (1) had a deletion larger than $25 \mathrm{bp}$ in relation to the reference; (2) had an insertion larger than $25 \mathrm{bp}$ in relation to the reference; and (3) mapped to two different locations with the 3' end of the read mapping $5^{\prime}$ of the $5^{\prime}$ end of the read (the pattern created by a tandem duplication). Because the nine genomes were sequenced at low coverage, our goal was not to identify all existing CNVs but instead to create a high-quality dataset of CNV breakpoints. To that effect, we applied a series of filters to minimize false-positive calls. Briefly, we required that each breakpoint was seen in at least two independent reads (from the same genome or from different genomes), that those two reads were not PCR duplicates, that the breakpoint was not located within the last $10 \mathrm{bp}$ of the ends of the reads and that the breakpoint mapped to the euchromatic region of the genome. We also excluded from the dataset all deletions/insertions that corresponded to transposable element polymorphisms (that is, the deleted/inserted sequence mapped exclusively to annotated transposable elements). Finally, we identified the exact breakpoint configuration by re-aligning the reads supporting each of the breakpoints to the reference genome sequence using Clustal [31,32].

Using this pipeline, we identified 447 deletions and 197 insertions larger than 25 bp segregating in the nine genomes. Because we required that at least two independent reads supported each breakpoint we biased our sample toward CNVs segregating in multiple genomes (as opposed to being private to one of the genomes). A total of $72 \%$ of $\mathrm{CNV}$ calls are supported by reads from at least two of the nine genomes, with only $28 \%$ of the CNVs supported by multiple reads from the same genome. This result is expected given the sparseness of the genomic data.

We evaluated the quality of our calls by confirming a subset of these variants by PCR and Sanger sequencing. Out of 32 CNVs tested, all were confirmed by PCR and sequencing. Sanger sequencing supported not only the existence of the CNVs but also the precise breakpoint configuration. We tested an additional set of eight CNVs that were filtered out from the final dataset because the reads supporting them were potential PCR duplicates. Again, all eight CNVs were confirmed, suggesting this was a fairly conservative filter. However, because our pipeline was able to identify a large number of CNV breakpoints $(n=644)$, and because our focus is on inference of mechanisms of $\mathrm{CNV}$ formation from sequence patterns using high-confidence $\mathrm{CNV}$ calls, we favored the more conservative dataset that minimized the number of false-positives.

To investigate the existence of potential differences between the mutational mechanisms underlying the formation of insertions and deletions, we used the $D$. simulans reference genome, and a parsimony approach, to polarize 
the calls (see Methods). Out of 447 deletions, 338 were confirmed to be deletions segregating in the sequenced $D$. melanogaster strains, 13 were re-classified as insertions in the reference genome, and 96 could not be polarized. Out of 197 insertions, 37 were confirmed to be insertions segregating in the sequenced $D$. melanogaster strains, with 123 being re-classified as deletions in the reference genome, and 37 could not be polarized.

Sizes of the identified insertions and deletions ranged from $25 \mathrm{bp}$ to $7.5 \mathrm{~kb}$, with a median size of $34 \mathrm{bp}$ and a mean size of $76 \mathrm{bp}$. The split-read method imposes no limit to the size of the deletions detected, but insertions are only detected if they are completely encompassed within a read. For this reason, the largest insertion detected in comparison to the reference genome sequence (that is, before polarization) was only $64 \mathrm{bp}$. Figure 1 shows the distribution of deletions, insertions, and unpolarized calls overlapping different functional contexts. Only nine of the $644 \mathrm{CNVs}$ (1\%) overlap coding exons: five are completely contained within the exon and four overlap both exonic and intronic sequence. All five $\mathrm{CNVs}$ located within coding exons have sizes that are multiples of three, suggesting they do not lead to frameshift mutations.

\section{Most insertions are not tandem duplications and correspond to de novo DNA}

After polarization, our dataset included 50 insertions: 13 present in the reference genome sequence and 37 segregating in the strains sequenced. Of the 50 insertions, only two (4\%) are tandem duplications, whereby the inserted sequence is a copy of a stretch of DNA already present in the genome (at a nearby location). Of the remaining 48 insertions, seven (14\%) correspond to simple expansions of dinucleotides or small repeats flanking the insertions, and $41(82 \%)$ have no match to the reference genome sequence and were thus classified as 'filler DNA' [13]. Filler DNA is a common outcome of the repair of DNA double-strand breaks by NHEJ in flies $[33,34]$ and other organisms [35]. Filler DNA has been observed in several studies of DNA repair that use artificial DNA constructs where DNA double-strand breaks are induced and the products of the DNA repair can be recovered and sequenced. In most cases, only a few nucleotides (or none) are added to the repaired junctions, but in some instances large insertions are created [13,33,35].

Filler DNA has been proposed to also include rearrangements of direct and inverted repeats located in nearby sequences [33]. We therefore investigated how much of each insertion classified as filler DNA could be attributed to both direct and inverted repeats present in its neighboring sequences. We considered four different window sizes to define neighboring sequences: $30 \mathrm{bp}, 60 \mathrm{bp}, 90$ bp, and 120 bp directly upstream and downstream from the insertion breakpoints. We then quantified the number of nucleotides in the insertions that matched neighboring sequences (see Methods). We also applied this procedure to a set of 41,000 simulated insertions that we created by shuffling the genomic coordinates of the actual insertions within each chromosome (retaining the insertions sizes). The goal was to determine how much overlap between a given stretch of DNA and its neighboring sequences is expected by chance. The boxplots in Figure 2A show the distribution of the proportion of nucleotides in insertions (and nucleotides in the simulated insertions) that match neighboring sequences. For the two smallest window sizes (30 bp and $60 \mathrm{bp}$ upstream and downstream from the insertions), the proportion of nucleotides in insertions that could be attributed to the copying of small stretches of DNA from neighboring sequences was significantly higher than what is expected by chance (Wilcoxon rank sum test, $P=0.002$ and

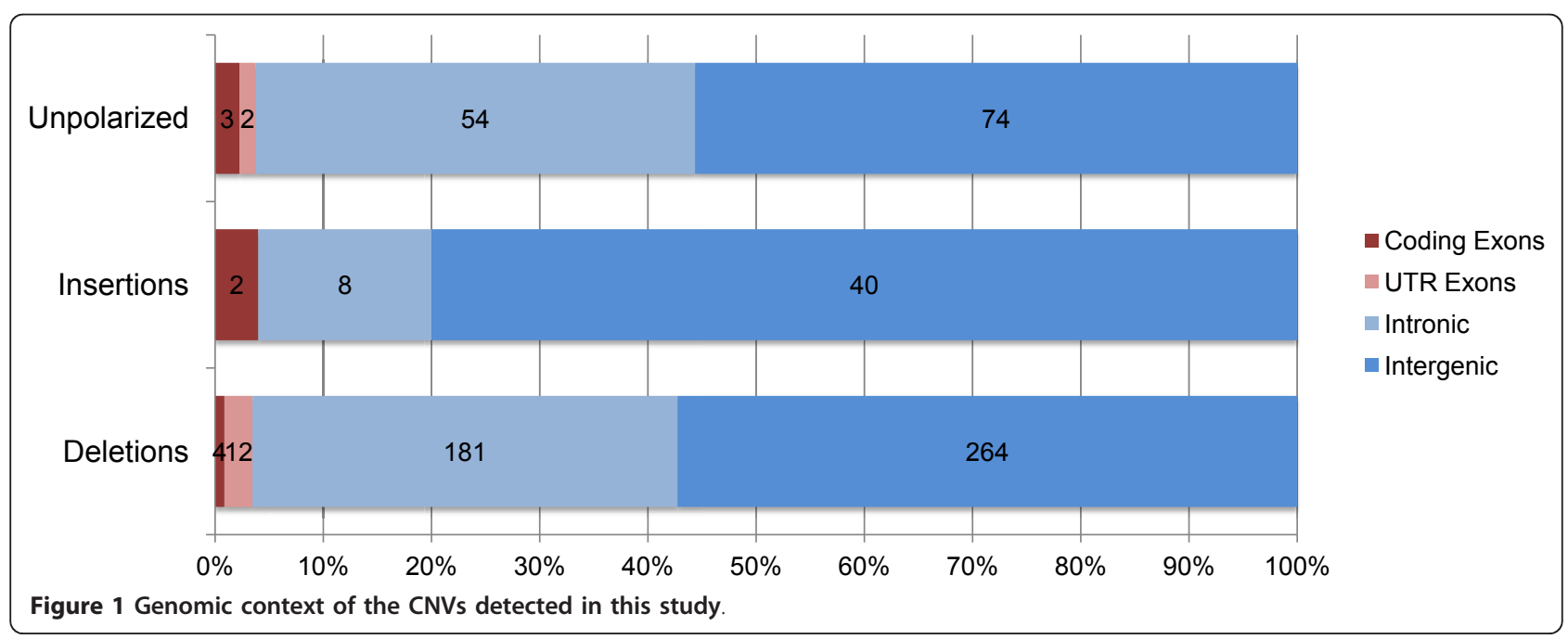




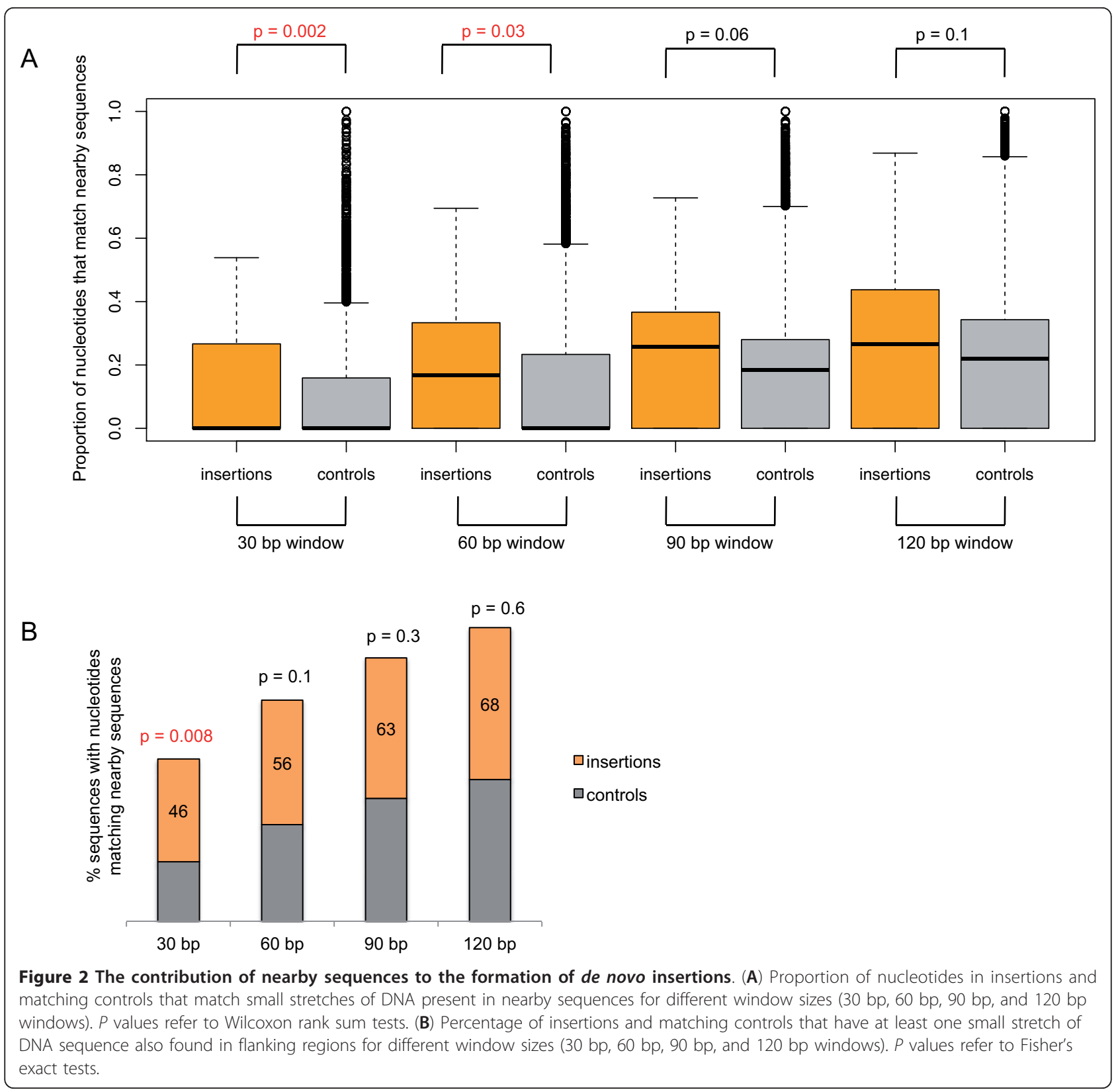

$P=0.03$, respectively). Accordingly, there is an excess of insertions with nucleotides matching neighboring repeats over the random expectation for the smallest window size (30 bp): 46\% of insertions have nucleotides that match repeats in neighboring sequences $v s .27 \%$ of random sequences (Fisher's exact test, $P=0.008$; Figure $2 \mathrm{~B}$ ). When larger window sizes are considered, a much larger fraction of insertions (and of nucleotides within those insertions) matches repeats in neighboring sequences. However, this is not different from what is observed for the set of simulated insertions (Figures 2A and 2B). Importantly, the matching repeats are typically small stretches of DNA (approximately 7 to $13 \mathrm{bp}$ ) and so even when present, they represent only a small fraction of the total number of inserted bases.

These data suggest that most insertions in D. melanogaster do not correspond to tandem duplications or to expansions of di- or tri-nucleotides or repeats, but instead that they are the product of the random incorporation of nucleotides and of the copying of small stretches of DNA from nearby sequences as part of the process of DNA repair. Although anecdotal, the fact that the two tandem duplications identified are also two of the largest insertions might be interpreted as suggesting that larger insertions may indeed correspond mostly to tandem duplications while smaller insertions (that is, smaller than $60 \mathrm{bp}$ ) will 
mostly correspond to novel stretches of DNA sequence. The observation that most insertions in Drosophila correspond to novel DNA sequence contrasts with a previous observation made for the human genome, where most recent insertions (1 to $100 \mathrm{bp}$; appeared after the humanchimpanzee split) were determined to correspond to tandem duplications [36].

\section{Distinct classes of CNV breakpoints}

The CNVs in our dataset fall into four breakpoint classes: (1) $41 \%$ have simple ends associated with small stretches of microhomology (minimum of $2 \mathrm{bp}$ ); (2) 35\% have blunt ends; (3) $22 \%$ have complex ends with additional nucleotides added or deleted to the breakpoint; and (4) $2 \%$ have complex ends (nucleotides added or deleted) and are also associated with stretches of microhomology (Figure 3). Microhomology is almost exclusively associated with simple ends, with only $5 \%$ of the breakpoints with microhomology also having additional inserted/ deleted nucleotides at the breakpoints. This result mirrors the observations made for human CNVs where only a minority of breakpoints with microhomology also had inserted/deleted nucleotides at the breakpoint (Table 1 $[19,20])$. There are no differences between insertions and deletions in the relative proportions of the four different types of breakpoints (Chi-square test, $P=0.54$ ).

The definition of what constitutes a breakpoint associated with microhomology differs across studies with some authors requiring only $1 \mathrm{bp}$ of identical sequence at the breakpoint (for example, [19]), while others require a minimum of 2 bp or more (for example, [20]). In order to determine the minimum number of identical nucleotides present at a breakpoint that are functionally relevant for $\mathrm{CNV}$ formation, we determined the number of nucleotides associated with three distinct types of microhomology for each of the 644 breakpoints in our dataset (Additional file 1, Figure S1). Microhomology of type I is the mechanistically-relevant form of microhomology associated with CNV formation: the deletion occurs between two sequences with microhomology such that one of the sequences becomes part of the deletion (the converse occurs in the case of an insertion). Microhomologies of types II and III (Additional file 1, Figure S1A) are not mechanistically associated with the formation of CNVs but can be used to determine the empirical expectation of finding a similar sequence of $n$ nucleotides close to the breakpoints by chance. As shown in Additional file 1, Figure S1B, only for $2 \mathrm{bp}$ or more do we find a significant excess of microhomology of type I versus the other two types (proportions test, $P=2.2 \times 10^{-12}$ ). As a result, in this study we required a minimum of $2 \mathrm{bp}$ of identical sequence to classify a breakpoint as showing evidence for microhomology. Of the 248 deletions associated with microhomology, only two have a stretch of microhomology $>20 \mathrm{bp}$. Thus, at most only two of the deletions in our dataset could have been created by SSA. This is likely to be an over-estimate because previous work in Drosophila has suggested that larger stretches of sequence identity are required to mediate SSA [37]. All other CNVs associated with microhomology consequently are either the product of NHEJ, MMEJ, or of replication-associated repair.

$\mathrm{CNV}$ breakpoints harboring complex ends (that is, additional bases present at the breakpoint) are significantly larger than CNVs associated with blunt ends, irrespective of the presence/absence of microhomology (median size of $43 \mathrm{bp} v s .32 \mathrm{bp}$, Wilcoxon rank sum test $P=1 \times 10^{-13}$ ). For 10 of 157 breakpoints with complex ends, the stretches of additional nucleotides inserted are large

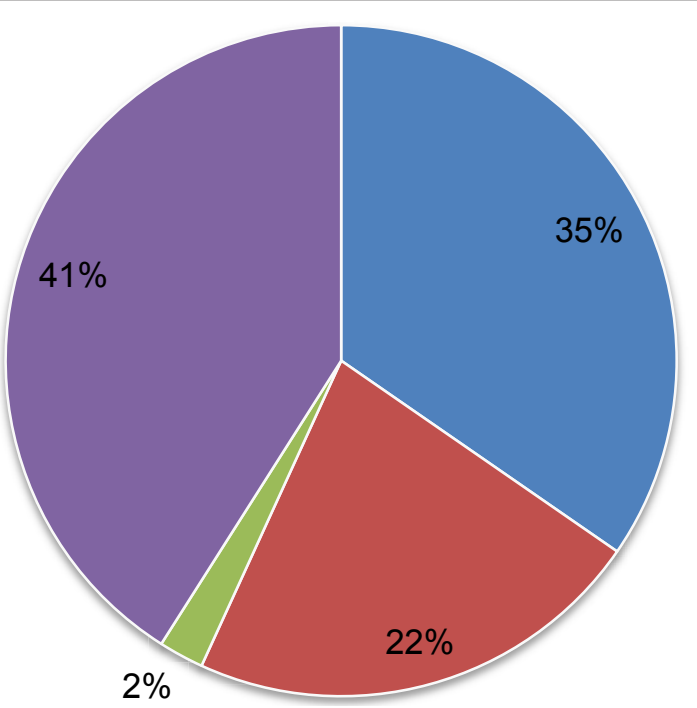

Blunt

- Inserted/Deleted nucleotides

- Microhomology and inserted/deleted nucleotides

- Microhomology

Figure 3 Distribution of CNVs among the different classes of breakpoints. 
Table 1 Comparison of the types of CNV breakpoints identified in Drosophila and humans.

\begin{tabular}{|c|c|c|c|c|c|c|c|}
\hline \multirow[t]{2}{*}{ Type of breakpoint } & \multicolumn{2}{|c|}{ Drosophila } & \multicolumn{2}{|c|}{$\begin{array}{l}\text { Human (Conrad } \\
\text { et al. })^{\mathrm{a}}\end{array}$} & \multicolumn{2}{|c|}{$\begin{array}{l}\text { Human (Kidd et } \\
\text { al. })^{\mathbf{b}}\end{array}$} & \multirow[t]{2}{*}{ Molecular mechanism(s) } \\
\hline & $n$ & $\%$ & $n$ & $\%$ & $n$ & $\%$ & \\
\hline Blunt & 223 & 35 & 58 & 19 & 82 & 11 & NHEJ \\
\hline Microhomology & 262 & 41 & 151 & 50 & 289 & 39 & MMEJ, replication-associated repair \\
\hline Blunt and large stretches of sequence identity ( ${ }^{3} 20 \mathrm{bp}$ ) & 2 & 0.3 & 3 & 1 & 219 & 29 & SSA, NAHR, replication-associated repair \\
\hline Inserted/deleted bases & 143 & 22 & 81 & 27 & 153 & 21 & NHEJ, replication-associated repair \\
\hline Inserted/deleted bases and microhomology & 14 & 2 & 9 & 3 & $3^{c}$ & 0.4 & MMEJ, replication-associated repair \\
\hline Total & 644 & & 302 & & 743 & & \\
\hline
\end{tabular}

The dataset of Conrad et al. refers only to deletions while the dataset of Kidd et al. includes both deletions and insertions. Excluded from both datasets of human CNVs were those variants classified as VNTRs (variable number of tandem repeats) and as transposable elements insertions. Further excluded from the dataset of Conrad et al. were 13 deletions that were also associated with inversions. Because Conrad et al. required only 1 bp of identical sequence to call a breakpoint as being associated with microhomology, we re-classified the entire dataset so that only deletions associated with at least 2 bp of identical sequence at the breakpoint were classified as being associated with microhomology.

enough (>20 bp) that they could potentially be mapped to the genome. If replication-based repair mechanisms are involved, the sequences of inserted bases are expected to map to the genome, often close to the deleted sequences. If NHEJ (or a form of alternative end-joining) is involved, the inserted bases should correspond to randomly inserted nucleotides and/or to rearrangements of repeats from nearby sequences (as seen for most insertions). There is no good genomic sequence match for any of the stretches of inserted bases. Furthermore, for seven of the 10 breakpoints, there are small stretches of identity between the inserted bases and nearby sequences that resemble the type of alignments seen between de novo DNA insertions and nearby sequences. These data favor the hypothesis that these CNVs are a consequence of NHEJ or alternative end-joining repair.

Table 1 compares the types of breakpoints identified in this study with those of two previous surveys of human CNV breakpoints $[19,20]$. There are two main differences between the types of breakpoints observed in Drosophila and in humans. The first is that in Drosophila there is a higher proportion of blunt breakpoints, a common outcome of NHEJ (35\% in Drosophila vs. $11 \%$ to $19 \%$ in humans). The second is that in Drosophila breakpoints are rarely associated with large stretches of high sequence identity, the hallmark of SSA and NAHR, while in humans Kidd and colleagues found that almost one-third of all breakpoints bore the hallmarks of these pathways [20]. As is clear from Table 1, the two surveys of human CNVs found a very different proportion of breakpoints potentially associated with NAHR (1\% in Conrad et al. vs. $29 \%$ in Kidd et al.). This difference is likely a consequence of the different experimental approaches used between the studies. Conrad and colleagues used a microarray capture strategy to identify the breakpoints of a subset of CNVs identified in a previous study [6], which may have biased their sample against CNVs associated with NAHR. Kidd and colleagues, on the other hand, identified CNV breakpoints using capillary sequencing of fosmid clone inserts, a powerful approach to sample the full spectrum of CNVs. Further support for a sizeable portion of human CNVs being associated with NAHR, comes from two other studies: one estimated that approximately $28 \%$ of breakpoints are associated with NAHR [22] and the other put it closer to $10 \%$ to $15 \%$ [6]. Motivated by the observation that different technological approaches can produce different results regarding the role played by NAHR in the formation of CNVs, we decided to re-analyze a dataset of 3,639 Drosophila CNVs identified using high-resolution tiling arrays [5] and determine if the observation that there is a paucity of Drosophila CNVs associated with NAHR is robust to the CNV detection platform used.

\section{NAHR plays a minor role in the formation of CNVs in Drosophila}

Emerson and colleagues [5] used tiling arrays covering the Drosophila genome at a resolution of 36 bp to identify 3,639 CNVs (2,211 duplications and 1,428 deletions) segregating in the genomes of 15 worldwide strains of D. melanogaster. Microarrays can only probe unique regions of the genome (that is, the probes in the microarray have to map to a unique genomic location), which means that they are biased against detecting additional duplications of regions of the genome that have already been recently duplicated. However, they are unbiased at detecting duplications of unique sequence where copy number changes from one copy (two copies in a diploid genome) to two copies (three or four copies in a diploid genome depending on the duplication being homozygous or heterozygous), irrespective of the presence/absence of flanking duplications. Therefore, we examined the breakpoints of these 3,639 CNVs for the presence of stretches of high-sequence identity in order to determine the contribution of homology-based mechanisms (such as SSA and NAHR) to the formation of CNVs in Drosophila. 
Unlike the 454 data, microarray data do not provide the exact breakpoint location. As a consequence, to look for the presence of stretches of high-sequence identity we considered the sequences 500 bp upstream and downstream the predicted CNV breakpoint and the CNV sequence itself. We looked for two types of sequence homology: (1) stretches at least $30 \mathrm{bp}$ in size with a sequence identity of at least $98 \%$ (type I; hallmark of SSA); and (2) stretches at least $200 \mathrm{bp}$ in size with a sequence identity of at least 95\% (type II; hallmark of both NAHR and SSA) (see Methods).

We found that only $2 \%(74 / 3639)$ of all CNVs were associated with sequence homology of type I (capable of mediating SSA), and 2.6\% (95/3639) with sequence homology of type II (capable of mediating SSA or NAHR). Because deletions in this dataset were associated with a high false-positive rate $(47 \%)$, we also restricted these analyses only to duplications (false-positive rate of $14 \%)$. Among the set of duplications, only $2.1 \%$ (46/2211) are associated with sequence homology type I, and 2.3\% (51/2211) with sequence homology of type II. Therefore, these results support the observation made using the 454 reads that homology-based mechanisms (SSA and NAHR) play a very limited role in the formation of CNVs in Drosophila.

Because both next generation sequencing and microarray technologies are biased against the detection of CNVs in non-unique regions of the genome (that is, segmental duplications and transposable elements) inferences about the importance of homology-based mechanisms are necessarily restricted to unique regions of the genome. However, unlike the human genome where segmental duplications and transposable elements can be found throughout the euchromatin, in Drosophila most repetitive elements are confined to the regions surrounding the centromeres (which have very low rates of recombination) with only a minority of these elements present in regions of the euchromatin with normal recombination rates $[26,27]$. Hence, our work suggests that outside of pericentromeric and telomeric regions, homology-based mechanisms play a minor role in CNV formation in Drosophila.

\section{Very high rate of CNV recurrence in Drosophila}

CNVs are classified as recurrent when different individuals carry independent but overlapping CNVs. The proportion of recurrent CNVs in the human genome has been estimated to be between $6 \%$ and $29 \%$ [6]. The sparseness of the 454 dataset prevents us from estimating from these data the proportion of recurrent CNVs in Drosophila. Therefore, in order to evaluate whether CNV recurrence is a common phenomenon in this taxon, we selected 26 genomic regions known to harbor at least one deletion in at least two strains based on the high-resolution tiling array dataset, and screened them in 15 worldwide strains by PCR and Sanger sequencing. These deletions are all located in the euchromatin, their mean size is similar to the mean size of the whole set of deletions and were predicted to range in frequency from 2 to 11 (median 2). Among the 26 regions, 12 (46\%) harbored more than one overlapping $\mathrm{CNV}$, suggesting a high rate of $\mathrm{CNV}$ recurrence in Drosophila.

Sanger sequencing of these 26 regions showed that the CNVs identified with the tiling arrays have identical characteristics to those identified with the 454 reads. There is no difference in the distribution of breakpoints types present in the 454 dataset and in the set of 36 CNVs (33 deletions and three insertions) segregating in the 26 regions described above (a total of $42 \mathrm{CNVs}$ were detected but for six (mostly tandem duplications) the breakpoints were not fully sequenced). In addition, similar to what was observed in the 454 dataset, CNVs with breakpoints harboring additional bases were, on average, larger than CNVs with simple breakpoints (that is, blunt ends with or without microhomology) (median $432 \mathrm{bp}$ vs. $211 \mathrm{bp}$, respectively; Wilcoxon rank sum test, $P=$ $0.005)$.

There was no difference in the distribution of breakpoint types between recurrent and non-recurrent CNVs. Furthermore, just as seen for the non-recurrent set, the recurrent CNVs were not associated with large stretches of sequence identity that might suggest their generation through NAHR. Instead, these data suggest that recurrent CNVs are mediated by non-homologous repair mechanisms. Among the 12 regions showing recurrent CNVs, three also show evidence for the presence of complex $\mathrm{CNVs}$. These occur when a single mutational event generates several breakpoints, that is multiple closely located CNVs segregating within the same individual. In these three regions the distance between distinct breakpoints ranged from $82 \mathrm{bp}$ to $325 \mathrm{bp}$. This association between complex CNVs (multiple CNVs within the same individual) and recurrent CNVs (multiple CNVs segregating in different individuals) suggests that some regions of the Drosophila genome are particularly unstable, and generate both complex events within individuals as well as independent but overlapping mutations between individuals. Though the sample size is small, these data suggest that complex CNVs may correspond to as much as $12 \%(3 / 26)$ of all Drosophila CNVs, a higher proportion than the 5\% estimated for the human genome [6].

\section{Non-B DNA structures are enriched in CNV breakpoints}

DNA conformations that do not correspond to the right-handed Watson-Crick double-helix are collectively termed non-B DNA $[38,39]$. These include sequences with Z-DNA motifs, quadruplex-forming motifs, inverted repeats, mirror repeats, and direct repeats [40]. 
Non-B DNA sequences have been found associated with the causal variants of several human diseases and have been proposed to cause genetic instability by impairing DNA repair and DNA replication [38,39]. Because errors during DNA repair and DNA replication are the ultimate causes of CNVs, we tested for the presence of non-B DNA sequence at the CNV breakpoints identified using the 454 data.

We focused on those variants that were detected by the presence of gaps in the reads of the sequenced genomes in comparison to the reference genome $(n=447)$ so that we could extract the CNV region and the flanking regions directly from the reference genome. For a control dataset, we shuffled the coordinates of the CNV breakpoints (25 bp within the CNV and the $200 \mathrm{bp}$ immediately flanking it 5 ' and $3^{\prime}$ ) randomly within chromosomes, so that there were 10 times more control sequences than CNV breakpoints. For both CNV breakpoints and control sequences, we identified non-B DNA sequences using the non-B DNA Motif Search Tool [40]. Figure 4 shows the distribution of non-B DNA sequences across the $200 \mathrm{bp}$ flanking the CNVs. In strong contrast to control sequences (in grey), which show a uniform distribution of non-B DNA sequences across their length, CNVs (in red) are enriched with non-B DNA sequences precisely at the breakpoints. Furthermore, there is a significantly higher number of $\mathrm{CNV}$ breakpoints (defined as the region spanning $25 \mathrm{bp}$ within the $\mathrm{CNV}$ and the $25 \mathrm{bp}$ immediately flanking it) associated with non-B DNA structures when compared to the control sequences: $11 \%$ vs. $5 \%$ (Fisher's exact test, $P=1.3 \times 10^{-5}$ ).

Some classes of non-B DNA sequences are more common than others (in both CNVs and control sequences), but for most we found a shift in the location of these repeats/motifs towards the $\mathrm{CNV}$ breakpoint when compared to the control sequences (Additional file 2, Figure S2), suggesting that most classes of non-B DNA sequences are associated with $\mathrm{CNV}$ formation. We found the non-B DNA sequences equally associated with the three classes of breakpoints (that is blunt breakpoints, breakpoints associated with microhomology, and breakpoints containing additional nucleotides inserted or deleted; Fisher's exact test, $P=0.98$ ). However, we found a significantly higher proportion of insertions associated with non-B DNA sequences than deletions (Fisher's exact test, $P=0.002$ ). The presence of non-B DNA sequences at a significant fraction of CNV breakpoints suggests a potential causal role for these sequences in $\mathrm{CNV}$ formation in flies.

\section{Discussion}

The detailed analysis of Drosophila CNV breakpoints suggests that non-homologous repair mechanisms are responsible for the formation of the majority of the variants. This is true not only for simple CNVs, but also for those that are recurrent and complex. We excluded a significant role for homology-based pathways (that is,

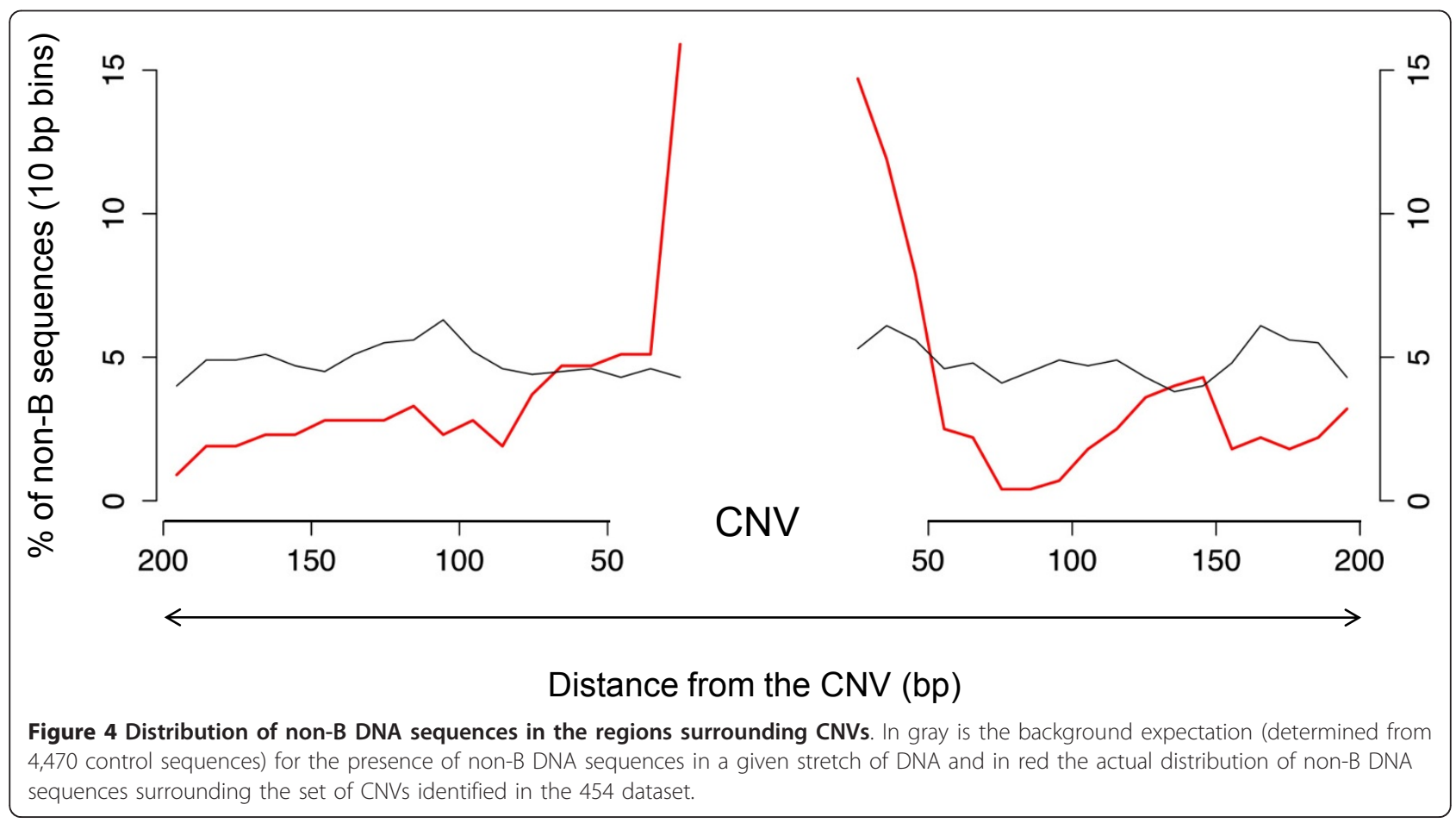


NAHR and SSA) in the formation of CNVs because only a minority of these variants (approximately 3\%) are flanked by stretches of high sequence identity. We also found little support for replication-associated mechanisms; the large stretches of additional nucleotides present at 10 breakpoints consisted of filler DNA, a result more consistent with NHEJ than with replicationassociated repair. The presence of microhomology at CNV breakpoints is, however, consistent with NHEJ, MMEJ, and replication-associated repair (for example, [41]). Determining exactly which pathway(s) are responsible for the different types of CNV breakpoints identified in our study will require the analysis of CNV breakpoints from fly mutants lacking the specific genetic requirements for each pathway (for example, [42,43]).

In the human and mouse genomes, NHEJ/MMEJ also underlie a large fraction of CNVs, though a sizeable fraction of CNVs are also mediated by NAHR (approximately $18 \%$ to $35 \%$ ) [19-22,44]. The difference in the preponderance of NHEJ/MMEJ in flies and mammalian genomes does not have to reflect intrinsic differences between these taxa in the relative usage of the different repair pathways (HR vs. NHEJ). In fact, NAHR and SSA are highly efficient in repairing DNA double-strand breaks in flies when these occur in artificial constructs flanked by repeats that can mediate these pathways [37]. Instead, the difference we observe between the taxa in the preponderance of homology-based mechanisms to the formation of CNVs likely reflects the different genomic architectures of the genomes: abundant and widespread presence of segmental duplications and transposable elements throughout mammalian genomes and less abundant and more restricted location (to pericentromeric and telomeric regions) of these elements in the Drosophila genome.

The Drosophila CNVs used in this work are significantly smaller than the published human CNVs. As a consequence, there is the possibility that some of the differences found between the two taxa may reflect different mutational mechanisms operating on CNVs of different size. We note, however, that in flies we found no differences between the breakpoints identified using the 454 data and those found using the high-resolution tiling array data despite the fact that the latter are significantly larger. Although the $\mathrm{CNVs}$ identified with the high-resolution tiling arrays are still smaller than those identified for the human genome, their size range already shows a significant overlap with that of the human CNVs used in this study. The absence of large CNVs in the fly genome likely reflects the much higher compactness of this genome (a much higher gene density means that large CNVs would overlap multiple genes) and the greater strength of purifying selection.

We have attempted to circumvent technical biases in CNV detection by making sure that our observations were robust to the platforms used to identify CNVs (that is, both next generation sequencing and hybridization based platforms). Still, our conclusions have to be necessarily restricted to the euchromatic sequence located outside of both pericentromeric and telomeric regions. The latter are highly enriched with transposable elements and segmental duplications making CNV detection extremely challenging irrespective of the platform used. The analysis of the CNV data generated by tiling arrays suggests both a high level of CNV recurrence and complexity (and coupling between the two) that can only be fully explored with technologies that are not biased against the detection of these classes of variants. The development of strobe sequencing technology and of ever larger reads [44] will greatly enhance this effort. By collecting a large number of breakpoints from a sparse low-coverage genomic dataset, we have demonstrated that the analysis of $\mathrm{CNV}$ breakpoints does not depend on high coverage datasets, instead read size is likely to matter the most.

Despite a minor role for NAHR, the fly genome is still punctuated by CNV hotspots $[9,10]$, that is, regions of the genome experiencing higher $\mathrm{CNV}$ mutation rates. These hotspots may share the properties of the mammalian CNV hotspots that are not associated with NAHR $[7,45]$. Unlike NAHR-mediated hotspots, where the reason for genomic instability is relatively well understood (that is, the presence of repeats leads to misalignments between homologous regions during DNA repair leading to further duplications/ deletions), it is not known what causes the instability associated with the remaining hotspots. One possibility is that these correspond to regions more prone to DNA breaks and/or that are harder to repair. In support of this hypothesis we do find that non-B DNA sequences, which are capable of impairing both DNA replication and DNA repair, are significantly enriched at $\mathrm{CNV}$ breakpoints.

One of the most surprising results stemming from this work is that in Drosophila most insertions correspond to de novo sequences. These novel stretches of DNA sequence are as large as $60 \mathrm{bp}$ in our dataset, potentially translating into the addition of 20 novel amino acids to a protein sequence. While it is likely that most of these insertions are deleterious, the occasional beneficial mutation could dramatically change the protein sequence in one single mutational event. This would allow very fast protein sequence evolution between closely related species. The frequent creation of novel stretches of DNA sequence as a consequence of DNA repair could have implications for the generation of genetic novelties and genome evolution in general.

\section{Conclusions}

Our results suggest that the different architectural features of the Drosophila and human genomes shape the mutation processes responsible for generating duplications, deletions, 
and insertions. Homology-based pathways contribute significantly more to the formation of CNVs in humans than Drosophila because of the abundance and widespread presence of segmental duplications and transposable elements in humans that can mediate HR. Instead, non-homologous repair is responsible for most CNVs in flies, including complex and recurrent CNVs. Non-homologous repair is also responsible for the creation of insertions made of de novo sequence, which have the potential to mediate rapid protein evolution. In addition, we show that non-B DNA sequences are enriched at $\mathrm{CNV}$ breakpoints, which makes these sequences good candidates for being associated with regions of higher $\mathrm{CNV}$ instability.

\section{Methods}

\section{Detection of CNV breakpoints in the 454 data}

Split-read methods were first applied to long Sanger sequencing reads [45] and CNVs were detected by identifying those reads which, when mapped to the reference genome exhibit a 'split' signature, either a gap in the reference genome (which suggests an insertion in the read, Additional file 3, Figure S3), a gap in the read (which suggests a deletion in the read, Additional file 3, Figure S3), or two sections of the read mapping to the genome with their positions flipped (which suggests a tandem duplication). Until recently, split-read methods were not widely used because of the small size of the reads produced by next generation sequencing platforms. Roche/454 technology is capable of generating reads $>100 \mathrm{bp}$ in size, however eukaryotic genome sequencing projects have predominantly relied on Illumina reads, which only recently achieved the 100 bp mark [46]. With these longer reads, split-read methods can readily identify with precise resolution the breakpoints of duplications, deletions, insertions, inversions, and translocations, as recently shown by the 1000 genomes project [21].

Sackton and colleagues used 454 technology to sequence at low coverage (approximately $0.2 x$ ) the genomes of nine $D$. melanogaster strains (three from an African population and six from a North Carolina population) [28]. We downloaded the original 454 reads (mean read size of $105 \mathrm{bp}$ ) from the Short Read Archive (SRP001156) and aligned them to the release 5 of the D. melanogaster genome using Mosaik (version 1.1.0021) [29]. We used the following Mosaik parameters to conduct the alignments: -hs 15 -mmp 0.05 -mhp 100 -act 26 -p 8 -bw 51. We discarded all reads that Mosaik mapped to the genome and kept only those that could not be mapped. We then used BLAT (version 3.4) [30] to map the latter reads. We ran BLAT using two sets of parameters: -fastMap and -oneOff $==1$. Finally, we detected CNV breakpoints with custom Perl scripts that parse the BLAT output and identify the split-read signature detailed in the Results section.
As discussed in the Results section, we applied a series of filters in an attempt to minimize the number of falsepositive calls. One of the filters applied was the removal of all CNVs supported exclusively by reads that could be PCR duplicates; these were defined as reads with the same exact start position but that could vary in their end position. Because of this filter all seven putative tandem duplications identified by our pipeline were excluded from the final CNV dataset. Another filter was the exclusion of all CNVs where at least $80 \%$ of the mutated sequence mapped to a known TE (TE annotation downloaded from FlyBase [47] release 5.29). After CNVs were identified, we re-aligned all supporting reads once again to the reference D. melanogaster genome using Clustal $[31,32]$ and those were the alignments used to classify the CNVs into the four classes of breakpoints. The CNV calls were polarized using the syntenic alignments between $D$. melanogaster and $D$. simulans [47]. If a deletion is called in one of the nine sequenced genomes but is also present (with similar breakpoints) in the $D$. simulans genome, then the most parsimonious explanation is that the variant is actually an insertion in the reference D. melanogaster genome (Additional file 3, Figure S3). Similarly, if an insertion is called in one of the nine genomes but a similar insertion is found in $D$. simulans, then the most parsimonious explanation is that we are detecting a deletion in the reference D. melanogaster genome (Additional file 3, Figure S3). $\mathrm{CNVs}$ were annotated (as exonic, intronic, and intergenic) using release 5.33 (retrieved from FlyBase [47]).

\section{Evaluating the contribution of nearby sequences to the formation of de novo insertions}

We used standalone Blast [48] (ncbi-blast-2.2.25) to identify stretches of high sequence identity between de-novo insertions and its neighboring sequences (for the window sizes defined in Results). We generated the random control sequences using BEDTools (shuffleBed; version 2.13.3) [49].

\section{Comparison of CNV breakpoints identified in Drosophila and human genomes}

Table 1 compares the types of CNV breakpoints identified in Drosophila and in two independent human datasets: one generated by Conrad and colleagues [19] and the other by Kidd and colleagues [20]. Both surveys of human CNVs included the identification of small tandem repeats (variable number tandem repeat (VNTR)) and of variants associated with the movement of transposable elements. Because studying these variants was not an aim of this work, Table 1 only refers to breakpoints of deletions and insertions. We also excluded from the dataset generated by Conrad and colleagues the 13 deletions (out of 315 ) that were also associated with inversions. Finally, as discussed in the Results 
section we only consider microhomology when there are at least $2 \mathrm{bp}$ of identical sequence present at the breakpoint. That meant re-classifying the breakpoints identified by Conrad and colleagues because they only required $1 \mathrm{bp}$ to classify a CNV breakpoint as being associated with microhomology (detailed breakpoint information was made available by the authors as Supplementary Material) [19].

\section{Evaluating the roles of NAHR and SSA}

To test for sequence identity shared between regions within CNV coordinates and flanking DNA, three sequence databases were generated for both the 454 and microarray data, using the reference Drosophila genome (version 5.27): (1) CNV sequence; (2) 5' flanking sequence; (3) 3' flanking sequence. The 454 data provide precise CNV breakpoints and, based on these coordinates, we extracted $200 \mathrm{bp} \mathrm{5'}$ and 3' of the CNV. The microarray data do not provide exact breakpoints, and for these data we defined the distal ends of the flanking sequences to be 500 bp 5' or 3' of the CNV coordinates. The proximal coordinates of the flanking sequences were set to extend $25 \%$ the length of the CNV 3' of the start of the CNV, or $25 \%$ the length of the CNV 5' of the end of the CNV. BLAT [30] (blatSuite.34) was used to search for sequence identity between: (1) 5' flanking sequence and the DNA within the CNV coordinates; (2) 3' flanking sequence and the DNA within the CNV coordinates; and (3) 5' flanking sequence and 3' flanking sequence. The data were filtered to return two datasets for each of these searches. The first filter was set to accept stretches of $\geq 30$ bp that possessed $\geq 98 \%$ sequence identity; the second was set to accept stretches of $\geq 200 \mathrm{bp}$ that possessed $\geq 95 \%$ sequence identity. The microarray results were further filtered to remove all 'self-hits' that resulted from the flanking sequences overlapping the $\mathrm{CNV}$ coordinates. Fasta files were generated for all sequences meeting the above criteria and were screened for repetitive sequences using RepeatMasker [50] (settings: abblast search engine, default speed/sensitivity, D. melanogaster annotations).

\section{Identification of recurrent and complex CNVs in the tiling array data}

We randomly selected 26 regions of the D. melanogaster genome that were identified by Emerson and colleagues as having deletions and that had been confirmed by PCR [5]. We screened these 26 regions in the same 15 natural populations analyzed by Emerson and colleagues. We identified recurrent CNVs by the presence of bands of different size (generated using the same pairs of primers) in different populations. We then sequenced these different bands by Sanger sequencing.

All statistical analyses were done using the statistical package $\mathrm{R}$ [51] and the application Rstudio.

\section{Data availability}

The Sanger sequences of the breakpoints of simple and complex CNVs initially identified using the tiling array data have been deposited in GenBank (KC138560KC138678).

\section{Additional material}

Supplementary Figure 1: Evaluation of the minimum number of
identical nucleotides present at the breakpoint that is required for
microhomology-mediated CNV formation. (A) Schematic
representation of the different classes of microhomology (type I refers to
the mechanistically relevant form of microhomology associated with CNV
formation). (B) Number of breakpoints showing $n$ identical nucleotides
for the three classes of microhomology.
Supplementary Figure 2: Distribution of non-B DNA motifs in
relation to CNV breakpoints. The beanplots in orange refer to
distribution of non-B DNA repeats in the sequences flanking the CNV
breakpoints (combines upstream and downstream sequences) while the
beanplots in grey refer to control sequences. The red line marks the
location of the CNV breakpoint (at position 25 bp of 225 bp of total
sequence). Small lines refer to individual observations (control sequences
have 10x more data) while the longer black line refers to the average of
the distribution. Each beanplot refers to a specific type of non-B DNA
motif.
Supplementary Figure 3: Description of the split-read approach
used to detect deletions and insertions and the rational for
polarizing the CNV calls.

\section{List of abbreviations}

CNVs: Copy number variants; FoSTeS: Fork stalling and template switching; HR: Homologous recombination; LCRs: Low-copy repeats; MMBIR: Microhomology-mediated break-induced replication; MMEJ: Microhomologymediated end-joining; NAHR: Non-allelic homologous-recombination; NHEJ: Non-homologous end-joining; SRS: Serial replication slippage; SSA: Singlestrand annealing; VNTRs: Variable number of tandem repeats.

\section{Authors' contributions}

All authors read and approved the final manuscript. MC-M, JRA, and AGC designed the study. MC-M carried most of the analyses with contributions from JRA. MC-M wrote the paper with contributions from JRA and AGC.

\section{Competing interests}

The authors confirm that they have no competing interests in the conduct of this research or preparation of this paper.

\section{Acknowledgements}

MC-M was supported by a postdoctoral fellowship from the Portuguese Foundation for Science and Technology (co-financed by POPH/FSE) and this work was supported in part by NIH grants R01 HG 003229 and R01 Al 064950 to $A G C$

Received: 31 May 2012 Revised: 25 October 2012

Accepted: 22 December 2012 Published: 22 December 2012

\section{References}

1. Zhang F, Gu W, Hurles ME, Lupski JR: Copy number variation in human health, disease, and evolution. Annu Rev Genomics Hum Genet 2009, 10:451-481.

2. Malhotra $D$, Sebat J: CNVs: harbingers of a rare variant revolution in psychiatric genetics. Cell 2012, 148:1223-1241.

3. Perry GH, Dominy NJ, Claw KG, Lee AS, Fiegler H, Redon R, Werner J, Villanea FA, Mountain JL, Misra R, Carter NP, Lee C, Stone AC: Diet and the evolution of human amylase gene copy number variation. Nat Genet 2007, 39:1256-1260. 
4. Cardoso-Moreira M, Long M: The origin and evolution of new genes. Methods Mol Biol 2012, 856:161-186.

5. Emerson JJ, Cardoso-Moreira M, Borevitz JO, Long M: Natural selection shapes genome-wide patterns of copy-number polymorphism in Drosophila melanogaster. Science 2008, 320:1629-1631.

6. Conrad DF, Pinto D, Redon R, Feuk L, Gokcumen O, Zhang Y, Aerts J, Andrews TD, Barnes C, Campbell P, Fitzgerald T, Hu M, Ihm CH, Kristiansson K, Macarthur DG, Macdonald JR, Onyiah I, Pang AW, Robson S, Stirrups K, Valsesia A, Walter K, Wei J, Wellcome Trust Case Control Consortium, Tyler-Smith C, Carter NP, Lee C, Scherer SW, Hurles ME: Origins and functional impact of copy number variation in the human genome. Nature 2010, 464:704-712.

7. Perry GH, Tchinda J, McGrath SD, Zhang J, Picker SR, Cáceres AM, lafrate AJ, Tyler-Smith C, Scherer SW, Eichler EE, Stone AC, Lee C: Hotspots for copy number variation in chimpanzees and humans. Proc Natl Acad Sci USA 2006, 103:8006-8011.

8. Cooper GM, Nickerson DA, Eichler EE: Mutational and selective effects on copy-number variants in the human genome. Nat Genet 2007, 39:S22-29.

9. Cardoso-Moreira MM, Long M: Mutational bias shaping fly copy number variation: implications for genome evolution. Trends Genet 2010, 26:243-247.

10. Cardoso-Moreira M, Emerson JJ, Clark AG, Long M: Drosophila duplication hotspots are associated with late-replicating regions of the genome. PLoS Genet 2011, 7:e1002340.

11. Gu W, Zhang F, Lupski JR: Mechanisms for human genomic rearrangements. Pathogenetics 2008, 1:4.

12. Hastings PJ, Lupski JR, Rosenberg SM, Ira G: Mechanisms of change in gene copy number. Nat Rev Genet 2009, 10:551-564.

13. McVey M, Lee SE: MMEJ repair of double-strand breaks (director's cut): deleted sequences and alternative endings. Trends Genet 2008, 24:529-538.

14. Zhang F, Carvalho CM, Lupski JR: Complex human chromosomal and genomic rearrangements. Trends Genet 2009, 25:298-307.

15. Quinlan AR, Hall IM: Characterizing complex structural variation in germline and somatic genomes. Trends Genet 2012, 28:43-53.

16. Lee JA, Carvalho CM, Lupski JR: A DNA replication mechanism for generating nonrecurrent rearrangements associated with genomic disorders. Cell 2007, 131:1235-1247.

17. Hastings PJ, Ira G, Lupski JR: A microhomology-mediated break-induced replication model for the origin of human copy number variation. PLOS Genet 2009, 5:e1000327.

18. Chuzhanova NA, Anassis EJ, Ball EV, Krawczak M, Cooper DN: Meta-analysis of indels causing human genetic disease: mechanisms of mutagenesis and the role of local DNA sequence complexity. Hum Mutat 2003, 21:28-44.

19. Conrad DF, Bird C, Blackburne B, Lindsay S, Mamanova L, Lee C, Turner DJ, Hurles ME: Mutation spectrum revealed by breakpoint sequencing of human germline CNVs. Nat Genet 2010, 42:385-4291.

20. Kidd JM, Graves T, Newman TL, Fulton R, Hayden HS, Malig M, Kallicki J, Kaul R, Wilson RK, Eichler EE: A human genome structural variation sequencing resource reveals insights into mutational mechanisms. Cell 2010, 143:837-847.

21. Mills RE, Walter K, Stewart C, Handsaker RE, Chen K, Alkan C, Abyzov A, Yoon SC, Ye K, Cheetham RK, Chinwalla A, Conrad DF, Fu Y, Grubert F, Hajirasouliha I, Hormozdiari F, lakoucheva LM, lqbal Z, Kang S, Kidd JM, Konkel MK, Korn J, Khurana E, Kural D, Lam HY, Leng J, Li R, Li Y, Lin CY, Luo R, 1000 Genomes Project, et al: Mapping copy number variation by population-scale genome sequencing. Nature 2011, 470:59-65.

22. Lam HY, Mu XJ, Stütz AM, Tanzer A, Cayting PD, Snyder M, Kim PM, Korbel JO, Gerstein MB: Nucleotide-resolution analysis of structural variants using BreakSeq and a breakpoint library. Nat Biotechnol 2010, 28:47-55.

23. Quinlan AR, Clark RA, Sokolova S, Leibowitz ML, Zhang Y, Hurles ME, Mell JC, Hall IM: Genome-wide mapping and assembly of structural variant breakpoints in the mouse genome. Genome Res 2010, 20:623-635.

24. Dopman EB, Hartl DL: A portrait of copy-number polymorphism in Drosophila melanogaster. Proc Natl Acad Sci USA 2007, 104:19920-19925.

25. Cridland JM, Thornton KR: Validation of rearrangement break points identified by paired-end sequencing in natural populations of Drosophila melanogaster. Genome Biol Evol 2010, 2:83-101.
26. Fiston-Lavier AS, Anxolabéhère D, Quesneville $\mathrm{H}$ : A model of segmental duplication formation in Drosophila melanogaster. Genome Res 2007, 17:1458-1470.

27. Bergman CM, Quesneville $H$, Anxolabéhère $D$, Ashburner $M$ : Recurrent insertion and duplication generate networks of transposable element sequences in the Drosophila melanogaster genome. Genome Biol 2006, 7 : R112.

28. Sackton TB, Kulathinal RJ, Bergman CM, Quinlan AR, Dopman EB, Carneiro M, Marth GT, Hartl DL, Clark AG: Population genomic inferences from sparse high-throughput sequencing of two populations of Drosophila melanogaster. Genome Biol Evol 2009, 1:449-465.

29. Mosaik Aligner. [http://code.google.com/p/mosaik-aligner/].

30. Kent WJ: BLAT - The BLAST-like alignment tool. Genome Res 2002, 4:656-664

31. Larkin MA, Blackshields G, Brown NP, Chenna R, McGettigan PA, McWilliam H, Valentin F, Wallace IM, Wilm A, Lopez R, Thompson JD, Gibson TJ, Higgins DG: ClustalW and ClustalX version 2. Bioinformatics 2007, 23:2947-2948.

32. Goujon M, McWilliam H, Li W, Valentin F, Squizzato S, Paern J, Lopez R: A new bioinformatics analysis tools framework at EMBL-EBI. Nucleic Acids Res 2010, 38:W695-699.

33. Ducau J, Bregliano JC, de La Roche Saint-André C: Gamma-irradiation stimulates homology-directed DNA double-strand break repair in Drosophila embryo. Mutat Res 2000, 460:69-80.

34. Rong YS, Golic KG: The homologous chromosome is an effective template for the repair of mitotic DNA double-strand breaks in Drosophila. Genetics 2003, 165:1831-1842.

35. Gorbunova V, Levy AA: Non-homologous DNA end joining in plant cells is associated with deletions and filler DNA insertions. Nucleic Acids Res 1997, 25:4650-4657.

36. Messer PW, Arndt PF: The majority of recent short DNA insertions in the human genome are tandem duplications. Mol Biol Evol 2007, 24:1190-1197.

37. Preston CR, Engels W, Flores C: Efficient repair of DNA breaks in Drosophila: evidence for single-strand annealing and competition with other repair pathways. Genetics 2002, 161:711-720

38. Cooper DN, Bacolla A, Férec C, Vasquez KM, Kehrer-Sawatzki H, Chen JM: On the sequence-directed nature of human gene mutation: the role of genomic architecture and the local DNA sequence environment in mediating gene mutations underlying human inherited disease. Hum Mutat 2011, 32:1075-1099.

39. Wang G, Vasquez KM: Non-B DNA structure-induced genetic instability. Mutat Res 2006, 598:103-119.

40. Cer RZ, Bruce KH, Mudunuri US, Yi M, Volfovsky N, Luke BT, Bacolla A, Collins JR, Stephens RM: Non-B DB: a database of predicted non-B DNAforming motifs in mammalian genomes. Nucleic Acids Res 2011, 39: D383-391.

41. Arlt MF, Rajendran S, Birkeland SR, Wilson TE, Glover TW: De novo CNV formation in mouse embryonic stem cells occurs in the absence of Xrcc4-dependent nonhomologous end joining. PLoS Genet 2012, 8: e1002981.

42. Chan SH, Yu AM, McVey M: Dual roles for DNA polymerase theta in alternative end-joining repair of double-strand breaks in Drosophila. PLOS Genet 2010, 6:e1001005.

43. Yu AM, McVey M: Synthesis-dependent microhomology-mediated end joining accounts for multiple types of repair junctions. Nucleic Acids Res 2010, 38:5706-5717.

44. Onishi-Seebacher M, Korbel JO: Challenges in studying genomic structural variant formation mechanisms: the short-read dilemma and beyond. Bioessays 2011, 33:840-850

45. Mills RE, Luttig CT, Larkins CE, Beauchamp A, Tsui C, Pittard WS, Devine SE An initial map of insertion and deletion (INDEL) variation in the human genome. Genome Res 2006, 16:1182-1190.

46. Mardis ER: A decade's perspective on DNA sequencing technology. Nature 2011, 470:198-203.

47. McQuilton P, St Pierre SE, Thurmond J, FlyBase Consortium: FlyBase 101the basics of navigating FlyBase. Nucleic Acids Res 2012, 40:D706-714

48. Altschul SF, Madden TL, Schäffer AA, Zhang J, Zhang Z, Miller W, Lipman DJ: Gapped BLAST and PSI-BLAST: a new generation of protein database search programs. Nucleic Acids Res 1997, 25:3389-3402. 
49. Quinlan AR, Hall IM: BEDTools: a flexible suite of utilities for comparing genomic features. Bioinformatics 2010, 26:841-842.

50. Smit AFA, Hubley R, Green P: RepeatMasker Open-3.0. 1996 [http://www. repeatmasker.org]

51. R Development Core Team: R: A Language and Environment for Statistical Computing. R Foundation for Statistical Computing. 2008 [http://www.R-project.org/].

doi:10.1186/gb-2012-13-12-r119

Cite this article as: Cardoso-Moreira et al:: Mutation spectrum of Drosophila CNVs revealed by breakpoint sequencing. Genome Biology 2012 13:R119.

Submit your next manuscript to BioMed Central and take full advantage of:

- Convenient online submission

- Thorough peer review

- No space constraints or color figure charges

- Immediate publication on acceptance

- Inclusion in PubMed, CAS, Scopus and Google Scholar

- Research which is freely available for redistribution 